\title{
DNA Damage and Repair in Human Spinal Cord Ischemia-Reperfusion Injury
}

Glen Roseborough*, Daqing Gao*, Ruxian Lin, Lei Chen, Yangseon Park, Eric Chu, G. Mel Williams, Chiming Wei

Cardiothoracic-Renal Molecular Research Program, Department of Surgery, Johns Hopkins University School of Medicine, Baltimore, MD 21205

*Equal contribution in this study.

During operations on extensive thoracoabdominal aneurysms, clamping of the aorta is likely to interrupt essential intercostal arterial blood supply to the spinal cord at least temporarily, leading to ischemia (1). This may mediate the generation of reactive oxygen species (ROS) on reperfusion and is likely the cause of post operative spinal cord dysfunction which remains a devastating complication (2-4). Animal models have been helpful in aiding our understanding of the molecular events attending ischemia and reperfusion of the spinal cord but questions always reside as to their relevance to man (5). In this report a post mortem examination after a tragic clinical outcome provided the opportunity to perform studies on the human spinal cord duplicating those we have reported in the rabbit (6).

A 69 year old man presented with documented expansion of a descending thoracic aortic aneurysm to $6.0 \mathrm{~cm}$. He succumbed from a stroke on the third hospital day following surgical repair. He also had bilateral leg paralysis. At autopsy done 4 hours after death there were remarkable differences between the thoracic or normally perfused spinal cord and the lumbar potentially ischemia or reperfused spinal cord. The measurements of injury were small in the thoracic spinal cord and extensive in the lumbar spinal cord DNA damage and DNA repair pathways. Figure 1 demonstrates the immunohistochemical staining of DNA damage products 8-oxoG, DNA base excision repair enzyme OGG1, DNA mismatch repair enzyme MYH and MSH2 in normally perfused thoracic spinal cord (control, C) and ischemia-reperfused lumbar spinal cord. These studies demonstrated that oxidative DNA damage product 8-oxoG was markedly increased in damaged spinal cord (D) compared with control spinal cord (C). The localization of 8-oxoG was in motor neuron of spinal cord. Furthermore, DNA repair enzymes, such as OGG1, MYH and MSH2 were also markedly increased in the damaged spinal cord compared with control spinal cord.

In conclusion, these findings duplicated those we reported in the rabbit subjected to $30 \mathrm{~min}$ of aortic occlusion and reperfusion injury (6). This is the first report in humans documenting DNA oxidative injury and apoptosis in ischemia-reperfusion injury of the spinal cord. This data suggest that DNA damage-repair system may play an important pathophysiological role in spinal cord ischemia-reperfusion injury.

\section{References:}

[1] P.N. Symbas, et al., J Thorac Cardiovasc Surg. 85 (1983) 300.

[2] L.G. Svensson, et al., J Thorac Cardiovasc Surg. 91 (1986) 71. 
[3] D.E. Carlson, R.B. Karp, and N.T. Kouchoukos. Ann Thorac Surg. 35 (1983) 58.

[4] K.D. Garlid, et al., Circ Res 81 (1997) 1072.

[5] S.H. Johnson, J.M. Kraimer, and G.M. Graeber. Stroke 24 (1993) 1547.

[6] R. Lin, et al., J Vasc Surg 37 (2003) 847.

[7] C. Wei, et al. Micros. Microanal.. 2 (1996) 786.

[8] This research was supported in part by the grant of NIH (HL61299) and Maryland Department of Health and Mental Hygiene.

Figure 1. Oxidative DNA damage product 8-oxoG and DNA repair enzymes MYH, OGG1 and MSH2 levels in control (C) and damaged (D) human spinal cord following ischemia-reperfusion injury [x1000].

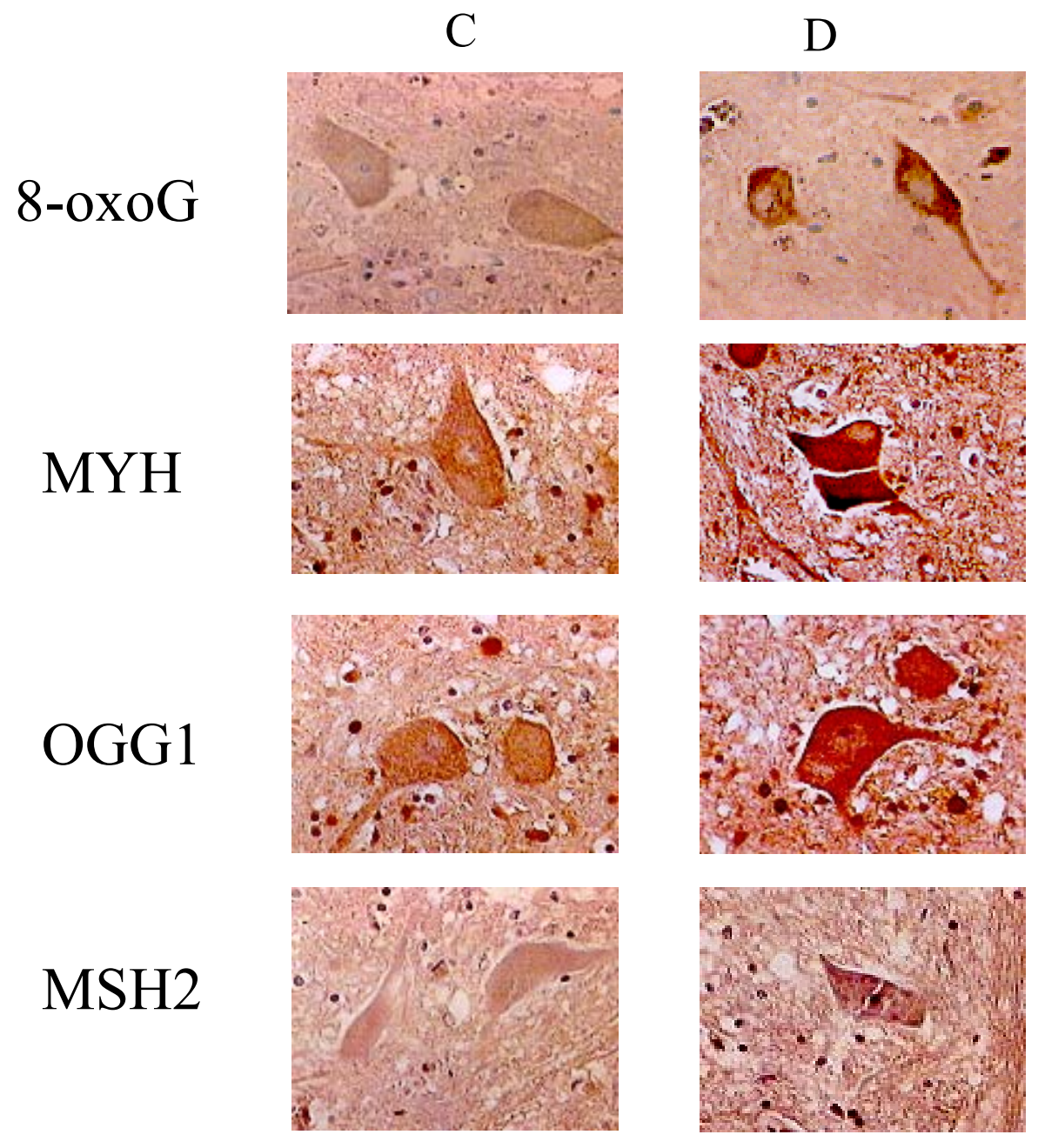

